\title{
A Tribological Simulation for a Typical Carbon Coated AFM Cantilever Interacting with a Monolayer Graphene Sheet
}

\author{
Kyriakos Grigoriadis ${ }^{1}$, Alexandros Palaiologos ${ }^{1}$, Anastasios Zavos $^{1}$, and Pantelis $G$. \\ Nikolakopoulos ${ }^{1 *}$ \\ ${ }^{1}$ Machine Design Laboratory, Dept. of Mechanical Engineering and Aeronautics, \\ University of Patras, Patras 26504, Greece
}

\begin{abstract}
This study aims on the dynamic and tribological characterization of a Single Layer Graphene Sheet (SLGS) including the effects of a graphene cantilever's deflection. A $10 \times 10 \mathrm{~nm}$ graphene model is developed, which is modally analyzed for both Zigzag and Armchair lattices. A typical Atomic Force Microscope (AFM) cantilever with carbon coated tip is also modeled during the simulation. The friction forces applied on the tip during its movement can be evaluated. The real contact area is characterized as the carbon atom tip is interlinked with the graphene atoms via the Lennard-Jones model. This study confirmed that the deformation of the AFM cantilever, is important to predict more accurately the tribological behaviour of graphene and the effect of its lattice orientation to its frictional properties. Therefore, this simulation provides an interesting way to understand the complex interaction between the cantilever tip and the sample in different contact conditions.
\end{abstract}

\section{Introduction}

Friction and wear are topics of great interest in many industrial and automotive applications such as high performance vehicles. Engine lubricants, combined with the surface characteristics, have a vital role in extreme contact conditions $[1,2]$. As a result of the high pressure and shear with reduced lubricant viscosity and ultra-thin films, the role of nanoscale mechanical testing has become crucial. Therefore, measuring in nanoscale requires great result accuracy.

Atomic force microscopy (AFM) [3] uses a microscope for materials characterization with a spatial resolution at a nanoscale level. The AFM sensor is a flexible micro-beam mounted on a chip with a sensor tip at its free end. Commercially available cantilevers inclusive chip are made of silicon single crystals by micromachining technology following some preferential cleavage planes. AFM and its several options have been used for obtaining high-resolution images of topography and other surface properties such as friction, electric, magnetic forces, mechanical stiffness, adhesion forces er.al. [4], probed by the cantilever tip in contact, intermittent contact, or non-contact with the sample by means of bending or torsion of the beam. The interaction forces between the cantilever tip and the examined specimen are dependent nonlinearly on the separation between them. For this reason, the modeling and analyzing of AFM dynamics are difficult. Thus, for a given set of parameters, contact mechanics and frictional characterisation of the cantilever tip are predominant in development of predictive methods.

By reviewing the most relevant studies in this area, it can be seen that the effect of capillary force on the dynamics motion along the sample requires great accuracy [5]. However, the fundamental question remains with respect to the design of a new AFM probe. Numerical

${ }^{1}$ Corresponding author: Pantelis G. Nikolakopoulos, e-mail: pnikolakop@upatras.gr 
simulation of vibrational resonances of stiff atomic force microscope cantilevers made of silicon by finite element methods (FEM) for application in contact-resonance spectroscopy was reported by Espinoza -Beltran et.al [6]. The FEM model considers the cubic symmetry of silicon single crystals and the geometrical shape of the cantilevers with a trapezoidal cross section and a triangular free end.

There have been several studies regarding the tribological characteristics of Graphene in the recent years. Bonelli et al. [7] simulated, via the tight-bonding atomistic method, the slipping of a deformable Graphene flake over graphite to reproduce actual AFM experiments. During these simulations significant stick-slip effect was observed as well as dependency of the tribological characteristics of graphene on the size, the stacking angle and the normal load applied in the graphene flake. Smolyantisky et al. [8] conducted a molecular dynamics simulation of the friction force microscopy (FFM) on suspended graphene in order to examine the relationship between graphene's friction and normal force under adhesive and repulsive regimes. For positive normal forces (repulsive regime) the friction force increased with respect to the normal force, however for negative normal forces (adhesive regime), as the FFM tip pulled the graphene sheet friction increased as normal force decreased. Recently, Palaiologos et al. [9] presented numerically the effect of a carbon tip with SLGS' dynamic and tribological behaviour, which can typically appear in nanoscale mechanical testing. Graphene has also shown significant interest due to its combination of potential mechanical, thermal, chemical and electrical properties. Nowadays, AFM deals with a wide variety of methods in which the probe interacts with the sample in different ways to characterize various material properties. AFM can characterize a wide array of mechanical properties (e.g. stiffness, friction), magnetic properties, and optical properties. Due to its ability and flexibility, the AFM is a common tool for material characterization having resolutions down to the nanometer scale and beyond.

The basic idea of this paper was the development of a simulation model in order to characterize monolayer graphene sheets, in terms of its dynamical and tribological behavior. Regarding the tribological characterization, a model is developed for virtual AFM measurements for the calculation of the static friction coefficient as a first step. The finite element method is used for the solution, using spring elements to simulate the interatomic forces and the beam flexibility. Beyond of the model's accuracy, the relative reduced computational time for the solution than the molecular dynamics for example is also another goal of the proposed model. The current study tries to simulate the AFMs basic operations, developing a virtual lab, and proceeds further on this issue by coupling the cantilever tip deflections on a Single Layer Graphene Sheet (SLGS). The model is also a generic model in the meaning that the tribo-contact materials can be changed, defining and investigating thus another pair of contact. All in all, a very reliable and quick virtual laboratory for measuring the static friction coefficient in atomic scale is proposed here.

\section{AFM Procedure Simulation}

The tribological model consists of an SLGS model recently presented by Palaiologos et al. [9] and an AFM cantilever model. Both models interact with each other via Van der Waals (VdW) forces acting between the cantilever tip and the SLGS atoms. For the simulation of the SLGS, the molecular structural mechanics approach of Li and Chou [10] is selected with some minor changes. For the AFM cantilever, we used a typical structural analysis beam model where at the tip, at its free end, a carbon atom is attached to simulate the tip coating. Both models interact with each other through elements which simulate the Lennard-Jones potential, allowing the calculation of the friction forces applied to the AFM tip. The VdW forces between the carbon atom of the cantilever's tip and the SLGS atoms are described by the Lennard-Jones theory. The combination of these three models simulated the Atomic 
Force Microscopy procedure by calculating the friction forces during the cantilever's tip movement over the graphene sheet. By utilizing this newly developed AFM model, we are able to examine the effect of the graphene chirality on its frictional behaviour.

\subsection{Modelling of the SLGS}

The model utilised for the simulation of the SLGS is the same with the one introduced by Li and Chou [10], which has already been used in numerous papers for the modelling of various carbon nanostructures, such as the carbon nanotubes and graphene sheets. In this model, the covalent bonds between the graphene's carbon atoms are represented by a circular crosssection beam element. Due to the negligible radius of the carbon nucleus $\left(r_{n}=2.7 \times 10^{-6} \mathrm{~nm}\right)$ compared to the carbon atomic radius $\left(r_{a}=0.067 \mathrm{~nm}\right)$ and the significantly smaller electron mass compared to the nucleus one can easily adopt concentrated masses to simulate carbon atoms. However, instead of using a consistent mass matrix or lumped mass matrix for the aforementioned beam elements, we selected the use of separate elements. Consequentially, in accordance with [11], we used separate mass elements for modelling the carbon atoms' masses. All the necessary parameters for our model are calculated in the Li and Chou approach [10] and are the same among other researches $[12,13]$ and are presented in Table 1.

Table 1. Basic element parameters for the SLGS model

\begin{tabular}{lcc}
\hline \multicolumn{1}{c}{ Parameter } & Symbol & Value \\
\hline Beam diameter & $\mathrm{d}$ & $0.147(\mathrm{~nm})$ \\
Young modulus & $\mathrm{E}$ & $5.488 \cdot 10^{-6}\left(\mathrm{~N} / \mathrm{nm}^{2}\right)$ \\
Shear modulus & $\mathrm{G}$ & $8.711 \cdot 10^{-7}\left(\mathrm{~N} / \mathrm{nm}^{2}\right)$ \\
Beam initial length & $\mathrm{L}$ & $0.1421(\mathrm{~nm})$ \\
Carbon mass & $\mathrm{m}$ & $1.9943 \cdot 10^{-26}(\mathrm{~kg})$ \\
\hline
\end{tabular}

For the current analysis, a 10x10 (nm) Single Layered Graphene Sheet is modelled. The dimensions of the graphene sheet are carefully selected in order to keep the computational cost of the finite element analysis (FEA), which is reviewed later, as low as possible and exclude the dimensional asymmetry from the factors affecting the SLGS' tribological behaviour. Therefore, we examined the sheer effect of the graphene's lattice chirality on its frictional properties. The SGLS model is positioned on a Cartesian coordinate system with the $\mathrm{X}$-axis and the $\mathrm{Y}$-axis coincident with the bottom and left edge of the sheet respectively, as shown in Figure 1. SLGS lattice can be categorized as zigzag or armchair based on the pattern of the atoms in the edge of the sheet. Therefore, moving along the $\mathrm{X}$-axis and $\mathrm{Y}$-axis corresponds to the zigzag and armchair lattice, respectively. 


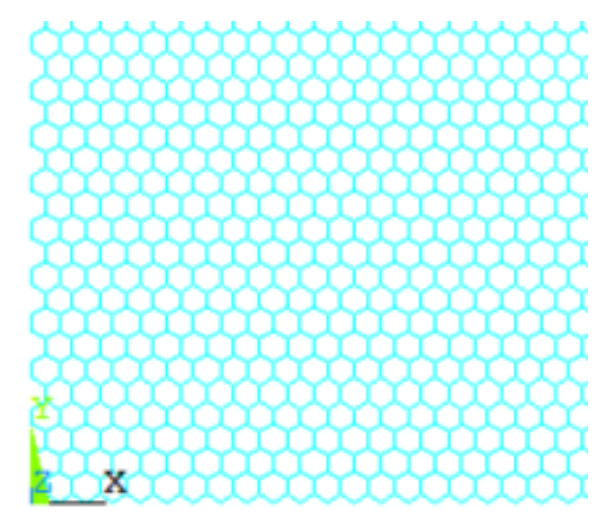

Figure 1. Preview of the SLGS distinctive beam-mass model.

\subsection{Modelling of the AFM cantilever}

The simulation of the AFM cantilever is a textbook example of the beam structural analysis. In this paper, a typical rectangular beam cantilever, made of Silicon, is modeled. Its dimensions are extracted from [14] while the mechanical properties of Silicon are reported in [15]. The cantilever data are presented in Table 2. Figure 2 shows a schematic representation of the AFM cantilever accompanied with its geometrical characteristics.

Table 2. Cantilever data

\begin{tabular}{lcc}
\hline \multicolumn{1}{c}{ Parameter } & Symbol & Value \\
\hline Cantilever width & $b$ & $30(\mu \mathrm{m})$ \\
Cantilever thickness & $h$ & $4(\mu \mathrm{m})$ \\
Cantilever length & $l c$ & $125(\mathrm{~nm})$ \\
Tip height & $l_{t}$ & $10 \div 15(\mu \mathrm{m})$ \\
Tip slope & $\varphi$ & $22.5\left({ }^{\circ}\right)$ \\
Si Young modulus & $E$ & $169(\mathrm{GPa})$ \\
Si Shear modulus & $G$ & $66.54(\mathrm{GPa})$ \\
Si poisson ratio & $v$ & 0.27 \\
Si density & $\rho$ & $2330\left(\mathrm{~kg} / \mathrm{m}^{3}\right)$ \\
\hline
\end{tabular}

Additionally, we assumed a carbon coating on the tip, which is simulated by a carbon atom on the AFM cantilever's tip. The model is fully customisable with the ability to simulate any model of the commercial rectangular AFM cantilever with a pyramid tip. 


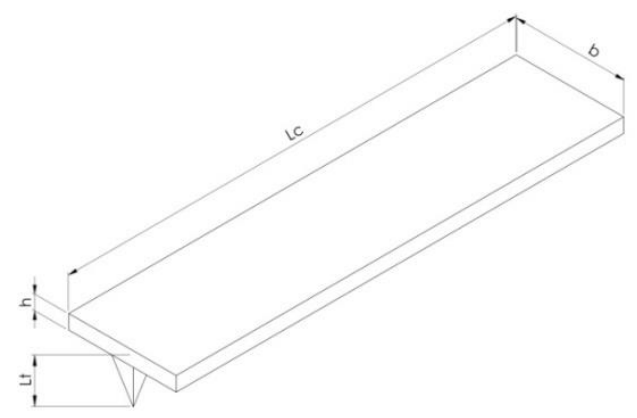

Figure 2. Schematic depiction of typical rectangular AFM cantilever

\subsection{Simulation of Van der Waals forces - Lennard-Jones theory}

Due to the proximity of the cantilever tip to the SLGS surface, forces are created and Van der Waals forces are believed to compose the majority of them. Those forces are modelled via Lennard-Jones "6-12" theory, as mentioned by Yazdchi et al. [16]. The Lennard-Jones potential due to the Van der Waals forces acting between the two carbon atoms over distance $r$ is described by Eq. (1), where $\varepsilon=0,38642 \cdot 10^{-12}(\mathrm{~N} / \mathrm{nm})$ and $\mathrm{r}_{0}=0,35(\mathrm{~nm})$ are the well depth and Van der Waals diameter for a carbon-carbon (C-C) atom pair, respectively.

$$
\mathrm{U}(\mathrm{r})=4 \varepsilon\left[\left(\frac{\mathrm{r}_{0}}{\mathrm{r}}\right)^{12}-\left(\frac{\mathrm{r}_{0}}{\mathrm{r}}\right)^{6}\right]
$$

The VdW force is calculated as the negative derivative of the Lennard-Jones potential, and is given in Eq. (2).

$$
\mathrm{F}(\mathrm{r})=-\frac{\mathrm{dU}}{\mathrm{dr}}=24 \frac{\varepsilon}{\mathrm{r}_{0}}\left[2\left(\frac{\mathrm{r}_{0}}{\mathrm{r}}\right)^{13}-\left(\frac{\mathrm{r}_{0}}{\mathrm{r}}\right)^{7}\right]
$$

The reason behind the minus in Eq. (4) is to sort the negative forces as attractive and the positive as repulsive forces. In Figure 3, the Lennard-Jones force is plotted for a range of distances between two carbon atoms.

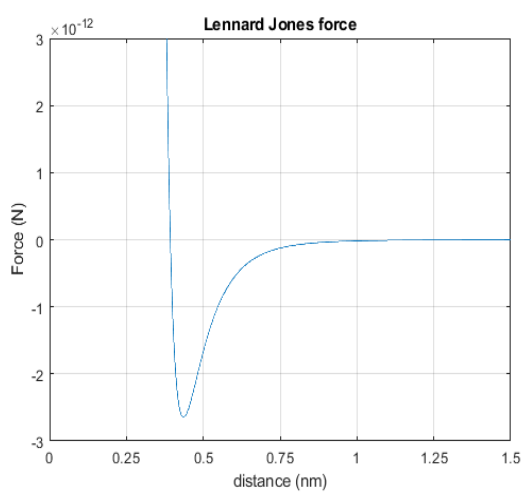

Figure 3. Lennard Jones force plot as function of distance between two carbon atoms. 
Figure 3 shows the strong repulsive forces at short range, indicating that the two atoms are strongly overlapping and repel each other. As the distance increases the repulsive forces get smaller and eventually turn into attractive forces, which reach a minimum value before they tend to zero at greater distances. At the distance where the transition from repulsive to attractive forces occurs the minimum potential energy is observed. The strong nonlinear behaviour of the Lennard-Jones forces is evident and determines greatly the solution algorithm that is adopted in this model.

\subsection{Tribological model}

For the tribological analysis the SLGS is placed on the XY plane as shown in Figure 1, where the zigzag and armchair lattice directions are parallel to the $\mathrm{X}$ and $\mathrm{Y}$ directions, respectively. The AFM cantilever is positioned above the SLGS with its one end, that where the tip is, suspended over the graphene sheet and the other end fixed in place. The carbon atom at the tip, which simulates the carbon coating, is connected with the SLGS atoms via spring elements with spring constants and pretension according to the Lennard-Jones theory. The pretension is given by Eq. (2), while the spring constant of these elements can be extracted by deriving the Lennard-Jones force equation and is given in Eq. (3).

$$
\mathrm{k}(\mathrm{r})=-24 \frac{\varepsilon}{\mathrm{r}_{0}^{2}}\left[7\left(\frac{\mathrm{r}_{0}}{\mathrm{r}}\right)^{8}-26\left(\frac{\mathrm{r}_{0}}{\mathrm{r}}\right)^{14}\right]
$$

Depending on the SLGS lattice whose tribological properties we are examining, the AFM cantilever is positioned accordingly, with its largest side being parallel to the lattice direction as depicted in Figure 4. Then the AFM cantilever is dragged along a path parallel to the lattice direction. In this instance the path runs parallel either to the $\mathrm{X}$ or the $\mathrm{Y}$ direction of the graphene sheet, to simulate the friction test on a zigzag or armchair lattice respectively. The path is discretised to 50 points and the reaction forces on the cantilever's fixed end are calculated at each point. The reaction force parallel to the path's direction is equal to the frictional force acting on the AFM tip, whereas the reaction force perpendicular to the XY plane denotes to the normal force acting on the tip. The ratio of the frictional force to the normal force gives the coefficient of friction during the AFM's movement along the path at each point.

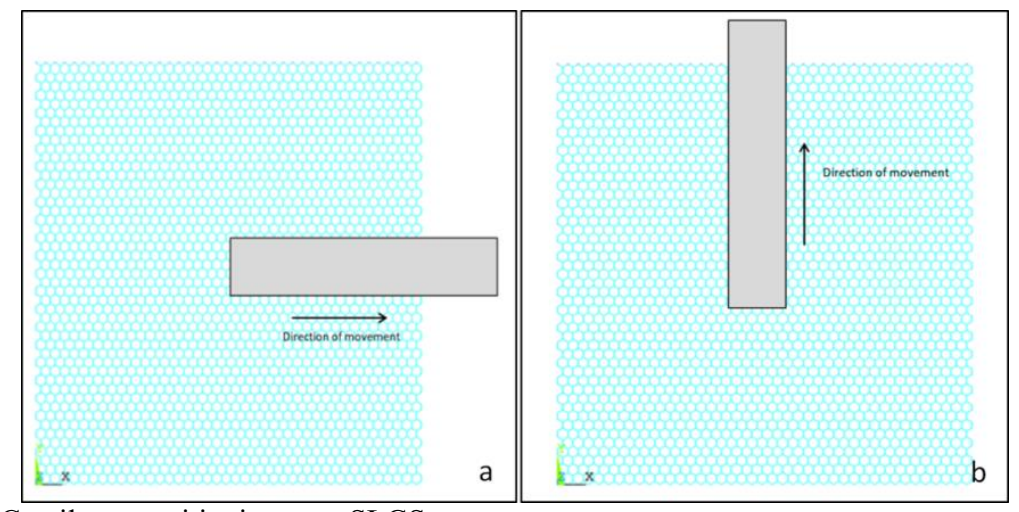

Figure 4. Cantilever positioning over SLGS

for zigzag (a) and armchair (b) lattice tribological analysis 


\section{Finite Element Modelling and Solution Algorithm}

The SLGS' complex carbon atom distribution is obtained by using the Nanotubes Modeler software. The coordinates created from the Nanotube Modeler are imported in ANSYS Multiphysics FEA software as nodes. We used two-node BEAM4 elements to simulate the covalent bonds of the SLGS atoms, and one-node MASS2 1 elements for the carbon atom masses. The tip's carbon atom also is represented by MASS21 element. The FE model of the AFM cantilever is comprised of BEAM188 elements which take into consideration the rectangular cross-section of the cantilever body, as well as the tapered square cross-section of the pyramid tip. The VdW forces between the tip and the SLGS carbon atoms are simulated by LINK11 elements. As previously mentioned, the Lennard-Jones force varies in a not linear way with the distance between atoms, therefore, the pretension and spring constant of each LINK11 element must also depend nonlinearly on its length according to Eq. (2) and Eq. (3), respectively.

To recreate the SLGS and substrate interactions all the peripheral graphene atoms are fully constrained, whereas the rest of them are allowed only to slide and to rotate on the XY plane. The LJ forces acting on both AFM tip and SLGS atoms caused them to deform thus altering the $\mathrm{C}-\mathrm{C}$ distance upon the $\mathrm{LJ}$ forces and spring constant have been previously calculated. This means that the carbon atom distances between the tip and the graphene should be recalculated by taking into account the displacements of the atoms. Because of this nonlinear $\mathrm{VdW}$ force behaviour, a pseudo-dynamic algorithm is adopted for the tribological model:

Step 1: At each point of the cantilevers path, for the initial position of the tip's carbon atom the distance between that and every SLGS atom is calculated. And a LINK11 element is created connecting these atoms. Depending on the element's length specific values of pretension and spring constant are attributed. A static analysis is performed to determine the displacement of the AFM tip and SLGS carbon atoms.

Step 2: The new distances between the tip and the SLGS atoms are calculated by taking into account the displacements of the atoms. If the new distances have changed by more than $10^{-}$ ${ }^{6} \mathrm{~nm}$ then new spring constants and pretensions are calculated for the new distances and a static analysis is performed to calculate again the displacements of both AFM and SLGS. This step is repeated until the change of new and old C-C distance is smaller than $10^{-6} \mathrm{~nm}$. When this criterion is met Step 3 of the algorithm is executed.

Step 3: The reactions on the fixed end of the cantilever are calculated. The friction force denotes to the reaction force parallel to the direction of the path while the static coefficient of friction equals to the ratio of friction force to the vertical reaction force on the cantilever. Then we moved to the next position of the path. Due to the static algorithm used to conduct the analysis the model calculates the static friction force and friction coefficient of the graphene sheet.

\section{Results and Discussion}

The results of the graphene tribological analysis can be summarized in Figures 5 and 6.The frictional forces and the coefficients of friction are presented for the zigzag and the armchair lattices, respectively. A comparison between these two cases can lead to some conclusions regarding the effect of chirality on the graphene's tribological behaviour. 

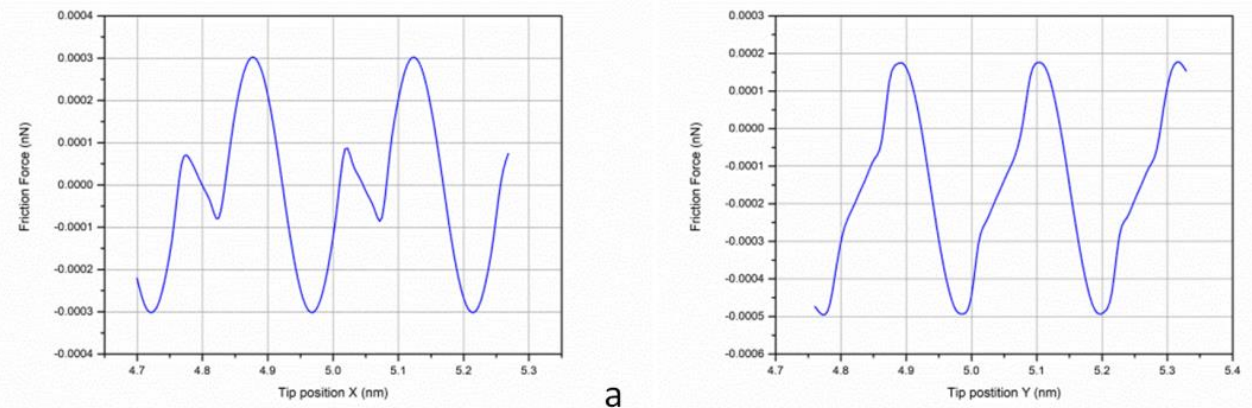

a

b

Figure 5. Static friction force of a) zigzag lattice and b) armchair lattice SLGS tribological analysis

An inspection of the friction force diagrams presented in Figure 5 can be really clarifying for the conditions and the mechanisms applied during the tip's movement over the SLGS. Both friction force plots show significant periodicity which is justified by the periodic distribution of the carbon atoms inside the graphene sheet lattice. The alternating sign of the friction force values is an indicator of strong stick-slip effects taking place during the tip's motion. The periodicity of the frictional forces agrees with the Prandtl-Tomlinson frictional mechanisms on the atomic scale. Apart from the different shape of the force graphs, it is also obvious that the friction force along the zigzag lattice direction (Figure 5 (a)) is evenly distributed around zero value (equal amounts of the force plot have positive and negative values). However, the friction force along the armchair direction (Figure $5(b)$ ) has higher negative values and lower positive ones (its average value is negative). This can be explained because the tip passes to the zigzag direction is impeded and aided equally, whereas in the armchair direction the tips movement is mainly hindered.

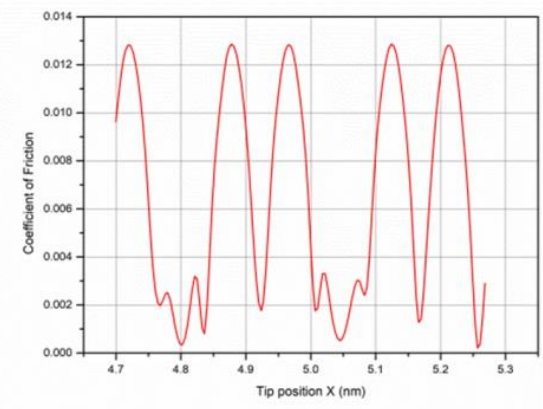

a

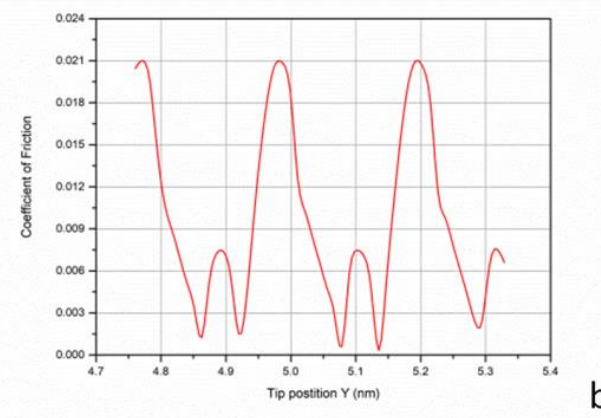

Figure 6. Static friction coefficient of a) zigzag lattice and b) armchair lattice SLGS tribological analysis

In Figure 6, the coefficient of friction (CoF) for the paths along the zigzag and armchair lattice is presented. Because in this current AFM model the normal force is varying depending on the tip's position over the SLGS, there is not an absolute analogy between the coefficient of friction and friction force diagrams. Despite their complex shape the graphs in Figure 6 provided useful information about the effect of the carbon atoms distribution to the graphene frictional properties. First, regarding the coefficient of friction of the zigzag lattice, its graph in Figure 6 (a) is periodic with maximum values around 0.0124 and an average $\mathrm{CoF}$ 
of 0.0066 . The graph consists of sections of large oscillations, when the tip is near to carbon atoms, and sections of much smaller oscillations near the minimum values, when the tips is at maximum distance from any carbon atom. Similar behaviour is observed for the CoF of the armchair lattice in Figure 6 (b) with large oscillations of its values near and smaller at greater distances from the carbon atoms. We found a periodicity in its values, however it has a maximum value of 0.021 , and an average $\mathrm{CoF}$ of 0.0096 which are around $69 \%$ and $45 \%$ higher than the zigzag $\mathrm{CoF}$ maximum and average values, respectively.

\section{Conclusions}

The main goal is to develop an accurate Atomic Force Microscopy virtual lab to gain an estimation of the expected results during the actual procedure. In this study a simulation model of the interaction between a typical AFM cantilever and a Single Layer Graphene Sheet was developed to evaluate graphene's tribological properties. The current model is able to recreate their behaviours more accurately by taking into account the deformations of both the AFM cantilever and the SLGS during the AFM path scanning procedure. It has successfully predicted the periodicity of frictional forces during the cantilever's movement over the graphene sheet which is also confirmed by the Prandtl-Tomlinson model. The effect of graphene's chirality to its tribological properties was examined by measuring the Friction forces and Coefficients of friction for an AFM cantilever traveling over the SLGS along both the zigzag and armchair lattice directions. The greater maximum and average values of the coefficient of friction as well as the domination of the "stick" phase of the stick-slip effect during the tip's movement along the armchair lattice showed that the zigzag lattice is more favorable for the cantilever's movement.

\section{References}

1. A. Zavos, P.G. Nikolakopoulos, Proc. IMechE, Part D: Journal of Automobile Engineering, 2017, 0954407017734770.

2. M. Mohammadpour, S. Theodossiades, H. Rahnejat and D. Dowson, Proc. IMechE, Part J: J. Engineering Tribology, 2017, 1350650117700756.

3. G. Binnig, C.F. Quate, C. Gerber, Phys. Rev., 56(9), 930-933, 1986.

4. Brukman, M.J. and Bonnell, D.A., Physics Today, p 36, June 2008.

5. R. Rasuli, A. Irajizad, M.M. Ahadian, IOP Publishing Ltd, Iran, 2010.

6. Espinoza-Beltrán, F.J., Geng, K., Saldaña, J.M., Rabe, U., Hirsekorn, S. and Arnold, W., 2009. New Journal of Physics, 11(8), p.083034.

7. Bonelli, F., Manini, N., Cadelano, E. and Colombo, L., 2009. The European Physical Journal B, 70(4), pp.449-459.

8. Smolyanitsky, A. and Killgore, J.P., 2012. Physical Review B, 86(12), p.125432.

9. A. Palaiologos, K. Grigoriadis, A. Zavos, P.G. Nikolakopoulos, presented at 6th European COnference on TRIBology,7-9 June 2017, Ljubljana, Slovenia.

10. C. Li, T.W. Chou. International Journal of Solids and Structures, 40(10), 2487-99,2003.

11. Hashemnia, K., Farid, M. and Vatankhah, R., 2009. Computational Materials Science, 47(1), pp.79-85.

12. Baykasoglu, C. and Mugan, A., 2012. Computational Materials Science, 55, pp.228-236.

13. Lengvarský, P. and Bocko, J., 2015. American Journal of Mechanical Engineering, 3(6), pp.225229.

14. Palacio, M.L. and Bhushan, B., 2010. Critical Reviews in Solid State and Materials Sciences, 35(2), pp.73-104.

15. Korayem, M.H. and Daeinabi, K., 2009, In Nanotechnology, 2009. IEEE-NANO 2009. 9th IEEE Conference on (pp. 896-899). IEEE.

16. Yazdchi K, Salehi M, Shokrieh MM. The International Conference on MEMS and Nanotechnology, ICMN'08,13-15 May 2008, Kuala Lumpur Malaysia. 